\title{
Training Robot Manipulation Skills through Practice with Digital Twin of Baxter
}

\author{
https://doi.org/10.3991/ijoe.v15i09.10493 \\ Igor Verner $\left.{ }^{\bowtie}\right)$, Dan Cuperman, Sergey Gamer, Alex Polishuk \\ Technion - Israel Institute of Technology, Haifa, Israel \\ ttrigoretechnion.ac.il
}

\begin{abstract}
This study explores an opportunity to engage first-year engineering students in practice with a modern industrial robot Baxter and provides training in spatial skills. We developed a laboratory exercise in which the students operate the robot to perform spatial manipulations of objects. We implemented the exercise on a digital twin of Baxter in the Gazebo virtual environment. The digital twin was calibrated to mimic the physical properties of the Baxter and correctly simulate its spatial manipulations with oriented cubes. The exercise was delivered to a class of 25 students as part of the robotics workshop in the Introduction to Industrial Engineering course. We administered a postworkshop questionnaire with focus on the analysis of the learning outcomes and students' spatial difficulties. The students noted that the workshop and particularly the exercise effectively exposed them to industrial robotics and raised their spatial awareness in robot operation.
\end{abstract}

Keywords-Virtual twin, Baxter, robot manipulations, first-year students.

\section{Introduction}

An industrial robot was traditionally defined as "an automatically controlled, reprogrammable, multipurpose manipulator programmable in three or more axes, which can be either fixed in place or mobile for use in industrial automation applications" [1]. This definition undergoes a radical change in the conceptual framework of the fourth industrial revolution (Industry 4.0), where a robot is considered as an essential component of a cyber-physical system (CPS) [2]. The two fundamentally new capabilities that extend the functionality of the traditional robots are: (1) internet communication for information exchange and collaboration with other devices of the CPS; (2) planning and taking actions, and interaction with humans based on intelligent technologies. Some of the basic functionalities that a modern autonomous robot should include are objects recognition, enhanced dexterity of spatial manipulations, and safe human-robot interaction.

The rapid development and growing deployment of CPSs prompts the need to include studies of Industry 4.0 technologies as integral part of engineering curricula. A number of courses on the subject have been developed in recent years [3]. Cheng [4] noted that while most of the courses target graduate students, there is a need to teach 
the subject in Bachelor programs. He developed an undergraduate upper-division course "Introduction to Cyber-Physical Systems" aimed to prepare students for creation and exploitation of such systems. Zalewski and Gonzalez [5] reported their Cyber Physical Systems course for undergraduate students majoring in software engineering. One of its educational objectives was to develop in students "awareness of the interactions of a cyberphysical system with the environment". Crenshaw [6] pointed that instructional robot platforms can be used to engage and motivate undergraduate students in experiential learning of the CPS key characteristics. In her course, the students created a networked system of different robots and devices working together to perform a collaborative task.

The intention of our research is to explore an opportunity to introduce basics of CPSs and modern robotics already from the first-year of engineering studies. The rationale is that early introduction to such concepts and practices can support students in their self-identification as future engineers, promote development of engineering and professional skills [7-9]. Our research proposes and investigates an approach which involves first-year engineering students in practice with a modern industrial robot Baxter and supports their training in spatial skills. The research was conducted in the robotics laboratory of the Technion Faculty of Industrial Engineering and Management. We engaged the students in planning and operating robots to execute complex spatial manipulations and evaluated the outcomes of this learning practice.

In the proposed approach the functionality of the modern industrial robot is studied through comparison with that of a previous generation robot manipulator. We introduce the students to both state-of-the-art and conventional technologies that they will meet in industry in the current transition period [10]. The goal is to impart not only technical skills needed to work with specific robots, but also the basic principles of robot programming and operation. Another goal is to organize students' practice with robots so that to foster their spatial awareness and train the spatial perception, mental operation and visualization skills. Studies indicate that learning practice in intelligent technology environments can be an effective way for cognitive training, and particularly, of visuospatial reasoning [11]. In the previous stages of research, we explored the approach in physical, virtual and remote environments based on conventional robot manipulators, using Scorbot 5 DOF and SCARA 4 DOF robots [12]. At the current stage presented in this paper we explore the opportunity to engage novice students in practice with the new generation industrial robot Baxter.

Baxter has a pair of 7 DOF arms and is designed to work safely and intelligently perform various production tasks in close proximity to people. The robot can adapt to its environment by sensing and controlling force, position, and torque at every joint, and by computer vision. Baxter serves as a platform for robotics research and advanced engineering education. Recently, experiments have begun on its use for educating novice students, e.g. first attempts to utilize Baxter as a robot-teacher in an elementary school class [13]. 


\section{Planning Robot Manipulations}

Planning robot manipulations is considered a complicated problem because of the need to select among a large number of possibilities for grasping and moving an object [14-16]. In this section we will discuss the aspects of the planning and the problems that students encounter when controlling a robot to manipulate objects.

\subsection{The rotation manipulation task}

The pick-and-place manipulation of an object is a basic task in robotics. It is discussed in literature, usually regarding one of the two main topics: grasp planning or path planning. While the former focuses on a stable grasping of the object, the later focuses on the robot's post-grasp trajectory. Nevertheless, grasping consideration should address not only stable grasping but also the possible need for object rotation during the manipulation $[14,15]$. This rotation is determined by the change in the orientation of the gripper between picking and placing the object.

To address the robot pick-and-place subject in a laboratory exercise for students, we selected a robot manipulation task in which the location and orientation of an object are defined in its initial and final positions. In this exercise, the students need to find within the reachable workspace of the robot the right way to grasp and rotate a simple oriented object i.e. a cube with symbols on its faces. To enable the students to easily define cube grasping and rotation, we developed a code language for such manipulations. In our previous studies $[12,17]$ we developed and used a limited code language which was tailored for a 5 DOF robot. For the current study we developed a new generic language suitable for cube rotation with each and every robot. This Rotation Manipulation Language (RML) is based on the notion that the cube is picked, rotated and then placed with a different orientation in its initial location. Thus, RML defines the initial orientation (while grasping the cube), and the final orientation (while ungrasping the cube). The user needs to consider grasp planning and ungrasp planning while the robot plans the path between the two. In other words, the rotation of the cube is a product of its change in orientation. RML relates to the world Cartesian coordinate system $(\mathrm{X}, \mathrm{Y}, \mathrm{Z})$ of the robot.
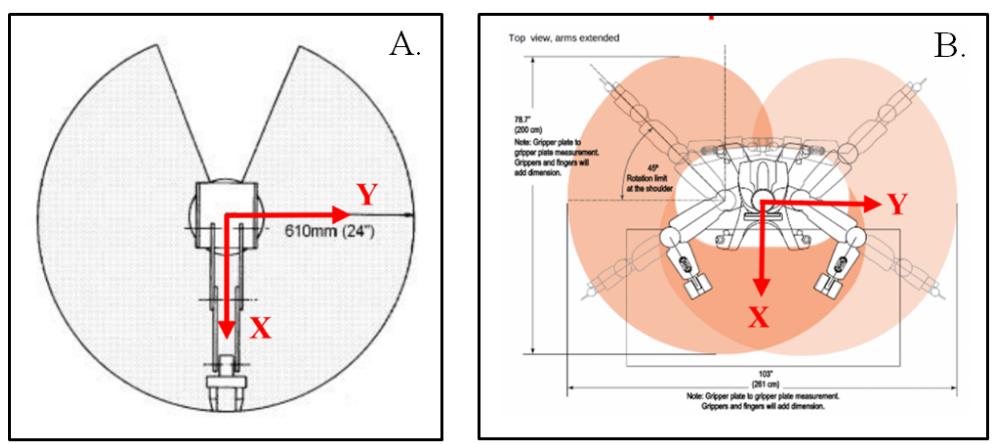

Fig. 1. Top views of the workspaces and world coordinate systems: 
A. Conventional robot Scobot [18]; B. New generation robot Baxter [19].

The coordinate system is defined for any robot so that the XY plane is horizontal and its origin is located at the center of the robot base, as in Figures 1A and 1B. The $\mathrm{X}$ axis is directed along the workspace symmetry line, towards the robot front workspace (see the top views). Manipulations are performed with the cubes placed in front of the robot and orthogonal to the coordinate axes.

The RML code consists of four characters in the format "S $\alpha \mathrm{F} \beta$ ". Here:

$\mathrm{S}$ - stands for the direction of the gripper axis when grasping a cube. It can get the values "X", "Y", or "Z", which refer to the positive directions of $\mathrm{X}$ and $\mathrm{Y}$ axes, and the negative direction of $Z$ axis.

$\alpha$ - determines the orientation of the gripper fingers when grasping the cube. It stands for the angle of rotation of the gripper around its axis. $\alpha$ and can get the values "2", "1", "0", or "-", which represent counter clock-wise rotations by $180^{\circ}, 90^{\circ}, 0^{\circ}$, and $-90^{\circ}$ correspondingly.

$\mathrm{F}$ - stands for the direction of the gripper axis when ungrasping the cube after the rotation, and can get the values $\{\mathrm{X}, \mathrm{Y}, \mathrm{Z}\}$ with the same meaning as $\mathrm{S}$.

$\beta$ - stands for the angle of rotation of the gripper around its axis, with the same meaning as $\alpha$.

In the example illustrated in Fig. 2A, the word "X0Z1" represent a rotation that starts with grasping the cube while the gripper axis is in line with $\mathrm{X}$ axis, and the gripper is not rotated around its axis. The cube is placed while the gripper axis is in line with the negative direction of $\mathrm{Z}$ axis, and the gripper is rotated $90^{\circ}$ around its axis. Fig. $2 \mathrm{~B}$ presents a robot Baxter arm in this position.
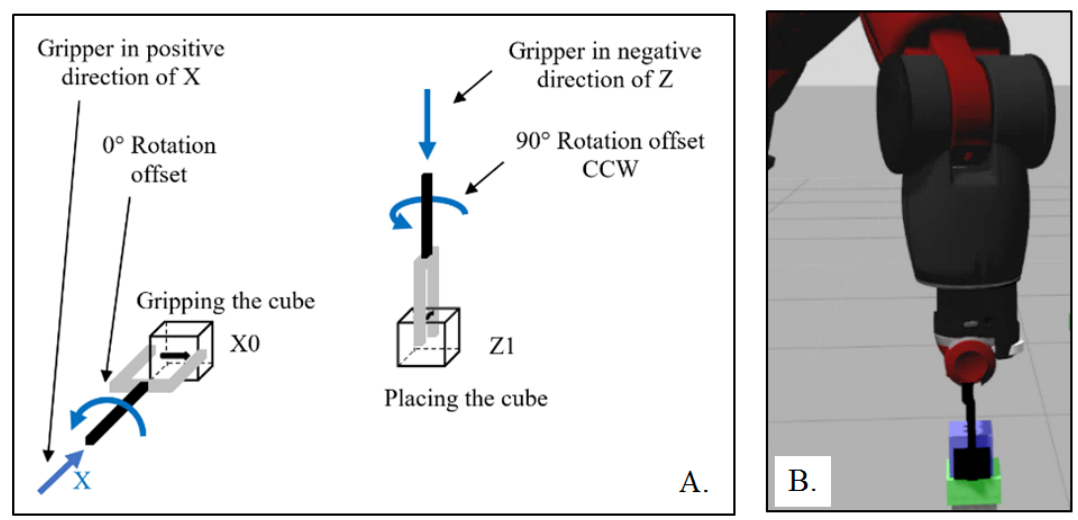

Fig. 2. A. The meaning of the rotation code "X0Z1"; B. Baxter in Z1 placing position.

Before picked or after placed, the cube can be in 24 different orientations, thus there are 576 orientation combinations. It can be shown that for all these combinations there are only 24 elementary rotations, by which the cube can be rotated from any initial to any final orientation. This can be done if the robot can approach and grasp the cube, which rests on the table, from all five possible orthogonal directions. 


\subsection{Rotation manipulations with robot Baxter}

The arm of the robot Baxter is presented in Fig. 3. It consists of seven joints marked with the following notations: Shoulder (S0 Roll, S1- Pitch), Elbow (E0 - Roll, E1- Pitch) and Wrist (W0 - Roll, W1 - Pitch, W2 - Roll).

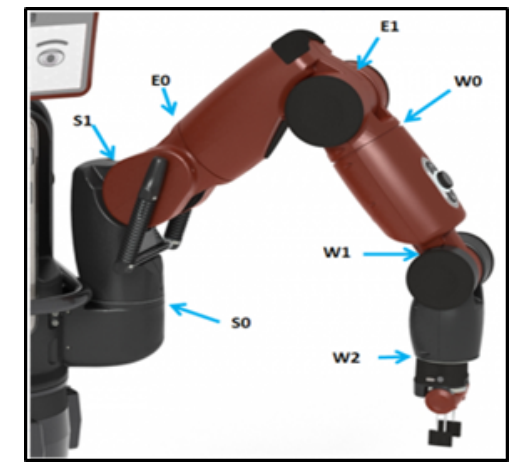

Fig. 3. The arm of the robot Baxter [20].

From experimentation with Baxter, we found that there are certain limitations in rotating a cube through a pick-and-place manipulation by a single arm. For instance, the cube, wherever placed, cannot be grasped from both positive and negative directions of the $\mathrm{X}$ axis. Also, choosing to approach the cube from both $+\mathrm{Y}$ and $-\mathrm{Y}$ directions significantly narrows the area where the cube can be placed. Therefore, in the exercise we excluded $-\mathrm{X}$ and $-\mathrm{Y}$ directions and programmed Baxter to handle the cube from three directions: $+\mathrm{X},+\mathrm{Y}$, and $-\mathrm{Z}$. So, not all 24 elementary rotations, described above can be achieved in the exercise with Baxter. Therefore, the cube cannot always be rotated from an initial to a final orientation in one pick-and-place manipulation. As Baxter is capable to grasp the cube from three directions, it can be shown that 19 out of 24 elementary rotations and 456 out of 576 manipulations of the cube are still possible.

\section{The Developed Workcell Environment}

\subsection{Baxter and its workcell}

The Baxter robot embodies core concepts of modern intelligent robotics. Thus, engaging novice engineering students in practice with Baxter could be of great value. However, Baxter is a complex engineering system that requires knowledge and skills far beyond that the novice students have. This fact raises the need for a simple user interface and an instructional strategy to make learning practice with Baxter accessible and effective for novice students. Baxter is operated and controlled via Robot Operating System (ROS) running in a Linux environment. Figure 4A presents a block 
diagram of the Baxter system in which the embedded computer uses ROS to receive feedback from the robot's sensors and to control limb actuators via motor controller.
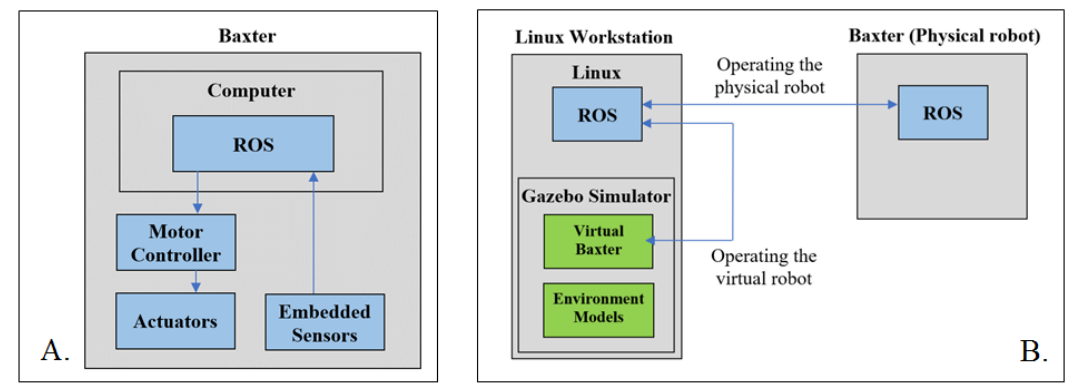

Fig. 4. A. Baxter's block diagram; B. User workstation system diagram.

The Baxter includes a software developers kit allowing users to develop custom software for the robot. The developed software can be run either on the physical robot or on its digital twin, within the Gazebo simulator, which models the robot and its environment. Figure 4B presents the diagram of the user workstation which can operate the physical and virtual robots. The Gazebo simulator can host the digital twin and models of objects in its environment.

For cube rotation exercise with the physical robot Baxter, we constructed two tables and placed them near the robot (Fig. 5A). A utility table on which we place the cubes needed for the exercise is located to the left of the robot. In front of the robot we located a second table to be used as a "buffer" on which a cube is placed for immediate rotation by the robot. The buffer was located in a spot in which the robot rotation dexterity is high.

For the exercise with the digital twin we developed a virtual workcell within the Gazebo simulator. We imported to Gazebo a virtual model of Baxter and added models of the cubes to be rotated and stands for placing the cubes (Fig. 5B). In this workcell, the user can choose the desired point of view by rotation the entire workcell and zooming in or out. Thus, the manipulated cube can be seen by default from a point of view of an observer in front of the Baxter (Figure 5B) or, for example, from a point of view of an observer standing in Baxter's place (Figure 5C).
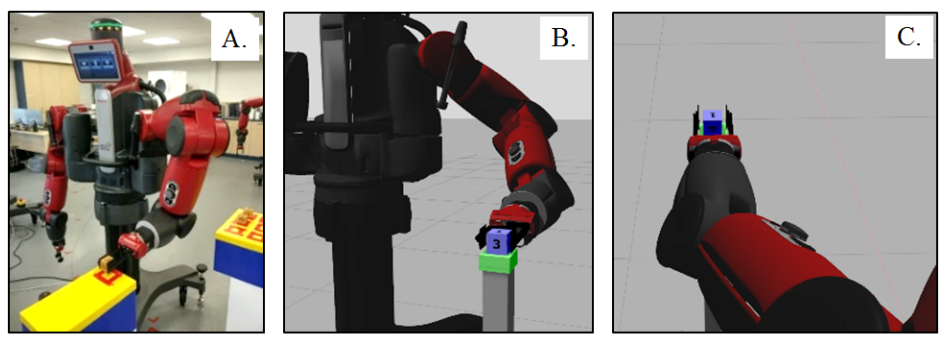

Fig. 5. A. Baxter places a cube; B. Virtual workcell; C Baxter's point of view. 
To rotate the virtual cubes, the user opens two Linux terminals: one for running Gazebo, and the other for controlling the digital twin by means of GUI we developed. While in a physical environment we start an exercise after placing real cubes on the utility table, in the virtual environment, the user needs to select the initial orientation of the virtual cubes. In the example presented in Figure 6, the initial orientation of the 3 cubes was defined by the digits 453 .
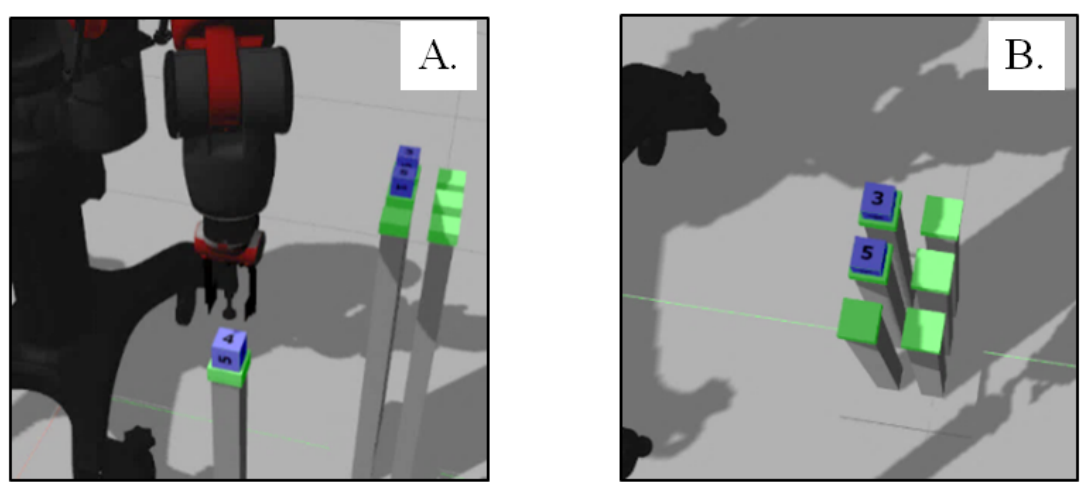

Fig. 6. A. Virtual cell: buffer (1), storage (2), destination (3); B. Cubes in the storage.

The user can set up the initial orientation of the cubes for the exercise by using the GUI presented in figure 7A. The orientations of the second and third cubes can be seen in Figure 6B. The first cube was moved to the buffer and can be seen in Figure 6A. Manipulating the cube is done by using RML with the robot control GUI presented in figure 7B. In the robot control GUI, selection of a cube number on the left side of the interface and pressing the button 'Take the cube to the buffer table' makes the Baxter pick up the designated cube from the utility stand and place it on the buffer stand. Setting the rotation arguments in RML and pressing 'Rotate the cube' makes the robot to perform the manipulation on the buffer. Pressing 'Put the cube to the utility table' makes the robot move the cube to the utility stand.
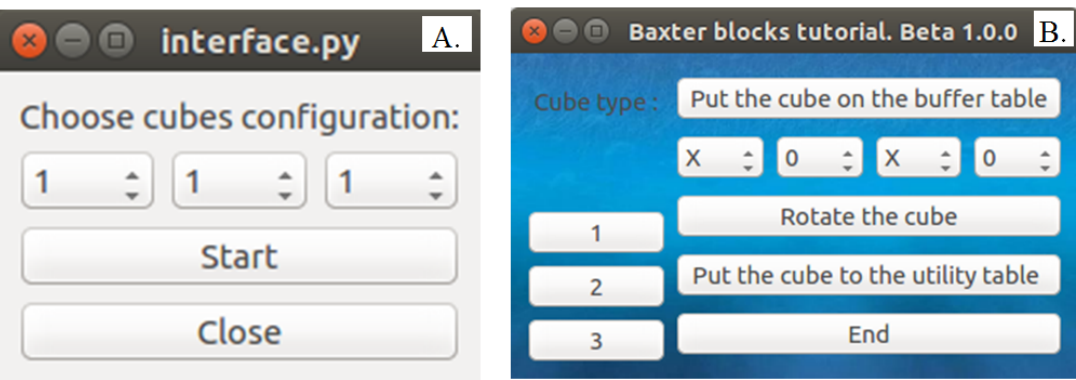

Fig. 7. The control interface: A. Configuration GUI; B. Robot control GUI. 


\subsection{Robotics workshop}

The 6-hour workshop was delivered to 25 first-year students participated in the Introduction to Industrial Engineering and Management course. The workshop included a lecture, and two exercises in operating of two different digital twins of robot manipulators. The lecture exposed the students to industrial robotics and introduced the concepts of robot operation in a workspace. The capabilities of the 7 DOF mechanical arm of Baxter in manipulating objects were discussed through comparison with that of the conventional 5 DOF vertical articulated arm of Scorbot.

The first preparatory exercise [12] was with the twin of Scorbot, operated in the robotic simulation environment RoboCell (Figure 8A). In the second main exercise, which was developed in this study, the students operated the digital twin of Baxter in Gazebo (Figure 8B). In both exercises the task was to manipulate three identical cubes with digits from 1 to 6 irregularly oriented on their faces, pick each cube in an initial orientation and place it in a desired orientation. The students ascertained that in some cases it is possible to find a suitable pick-and-place operation which implements the desired manipulation of the cube, while in other cases they need to plan a sequence of two operations.

The orientations of the cubes in the tasks were designed so that the students could evaluate the dexterity differences between the two robots. The desired orientation of the first cube could be achieved in a single pick-and-place operation with either the Scorbot or the Baxter. The manipulation of the second cube could be executed by one pick-and-place operation of Baxter but required two operations of Scorbot. The manipulation of the third cube required at least two pick-and-place operations from both robots.
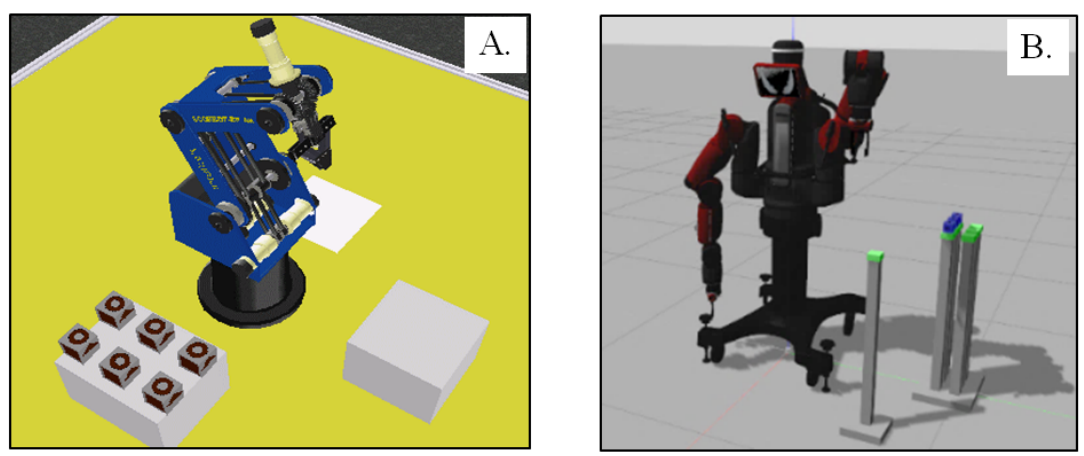

Fig. 8. A. Scorbot RoboCell; B. Baxter's virtual workcell.

In the Baxter exercise, the students used the GUI, described in the previous section, to pick a cube, move it from the storage area to the buffer, rotate it to the desired orientation, and place it in the destination position at the assembly area. The students worked in pairs and were given a time limit of 45 minutes to complete the exercise. During the exercise we noticed that some students took advantage of the opportunity to change workcell perspective as described in the previous section. Some students 
used the default perspective of an observer looking at the Baxter from the front (Fig. 5B), while some decided to change it and preferred Baxter's point of view (Fig. 5C). The students were offered a physical cube to help them in planning robot manipulations. Some of the students used the cube, while some others imitated movements of the robot gripper with their hand (Fig. 9). Some didn't use any physical objects and planned the rotations mentally in their heads.

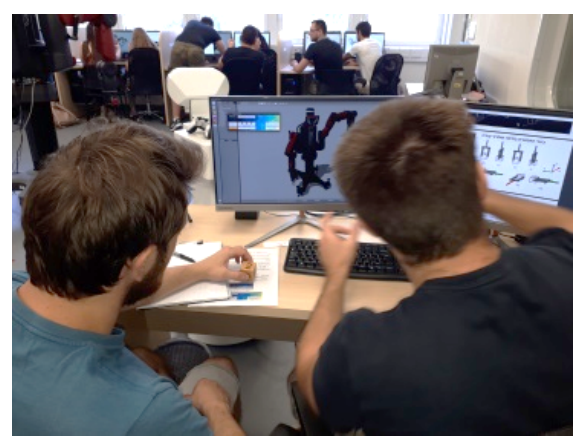

Fig. 9. Students perform the exercise.

\section{$4 \quad$ The Study}

Our study aimed to evaluate learning outcomes of the workshop with special attention to the exercise with the Baxter's digital twin. We inquired how the workshop influenced students' understanding of the role of robotics in modern manufacturing, and their interest in the subject as future engineers. We asked whether and how the practice in operating the digital twin exposed the students to industrial robotics and contributed to their spatial awareness in robot operation. We also inquired into the spatial difficulties that the students faced when they performed rotation manipulations in the virtual robot cell.

\subsection{Data collection and analysis}

In this study, two post-workshop questionnaires were administered. The first one requested students' reflections on the workshop experience. We asked the students if the workshop exposed them to industrial robotics and raised their awareness of spatial problems in planning and operating robot manipulations. Responses were accepted from 25 students. Results of the questionnaires were analyzed and triangulated with the evidence elicited from the laboratory reports and observations.

The second questionnaire was about the spatial difficulties faced by the students when they performed the exercise with the digital twin of Baxter. The students were asked to evaluate the level of difficulties in using the rotation manipulation language of spatial codes to find optimal sequences of rotation manipulations to perform the robot task. An additional question was about the difficulties in applying the exocentric view of the workspace from the robot's view point. When performing the exercise, 
some students, who had spatial difficulties, upon request got physical cubes, same as that rotated by the robot, and rotated them with their hands, to make it easier to imagine manipulations by the robot. We asked those students, to what extent the manual rotations helped them to plan the robot manipulations.

\subsection{Findings}

Workshop contribution: From the results of the first post-workshop questionnaire, most of the students did not study robotics and had no experience with virtual or real robots before the workshop. Their evaluation of the workshop's contribution was highly positive:

- $86 \%$ reported that the workshop exposed them to industrial robotics,

- $62 \%$ noted that the workshop was useful and relevant to their future profession,

- $43 \%$ pointed that the workshop contributed to their understanding of basic concepts in the field of industrial robotics.

- $71.5 \%$ reported that performing the exercise on a digital twin of Baxter contributed to their understanding about spatial skills required to operate robot-manipulator.

We found moderate Pearson correlations between the above-mentioned contribution factors. Statistically significant positive correlations were found for the awareness of spatial problems and for the exposure to industrial robotics $r=0.52(p<0.05)$ as well as between the relevance to future profession and the awareness of spatial problems $\mathrm{r}=0.8(\mathrm{p}<0.0001)$.

The highly positive evaluation of the learning practice is expressed also in students' reflections. The repeated reflections related to exposure to industrial robotics:

"The lecture and the labs exposed me to industrial robotics, the subject I had never heard before. It gave me general knowledge of this profession and a taste of experience into subject. This exposed me also to a variety of robots and their capabilities."

"An industrial engineer needs to know new worlds because everything can be relevant to his field of competence, especially technology and robots. Exposing such subjects will help him in the future profession. I was interested in the robot-twin. The way to operate it related to different aspects such as angles, point of view, and rotation commands."

The workshop helped students understand the value of industrial robotics for their future profession:

"In my opinion as future industrial engineers, we had to know how to interact with machines and robots, and this was a learning experience."

The students noted their progress in spatial skills for robot operation:

"The experience with the robot contributed to my spatial skills and exposed me to the wide range of robot movements that can be performed with minimum number of commands."

Spatial difficulties in manipulating objects with the digital twin: In the second post-workshop questionnaire the students noted spatial difficulties that they experienced when performing the exercise. In particular, $65 \%$ of them noted difficulties in using the rotation manipulation language, $61 \%$ in finding optimal sequences of rotation manipulations and in considering the workspace from the exocentric point of 
view. $87 \%$ of the respondents noted that the opportunity to rotate the oriented cube by hand significantly helped them to overcome the spatial difficulties and successfully complete the exercise.

\section{Conclusion}

Our research explores ways to introduce industrial engineering students at the firstyear of their studies to the concepts and technologies of industrial robotics. In the previous stages of research, we developed and conducted a workshop in which the students controlled and operated a conventional robot manipulator Scorbot in physical, virtual and remote environments, to manipulate objects in the workspace.

In the current study we developed an exercise in control and operation of a new generation robot Baxter. We conducted a workshop which included a preparatory exercise with the digital twin of Scorbot and the new exercise with the digital twin of Baxter. In both exercises, the students controlled and operated robots to execute complex spatial manipulation tasks that require translation and rotation of oriented objects (cubes) in the workspace. We developed a language of spatial codes to describe pickand-place manipulations for different rotations of the cube. The students used the language for planning robot manipulations. The language also helped them to explore and compare the dexterity of the conventional and modern robots.

The experiments in using new generation industrial robots such as Baxter in education of novice students started very recently. For our knowledge, the developed exercise and the workshop for first-year industrial engineering students are the first of their kind. The workshop experience showed that despite the mechanical and software complexity of robot Baxter, its virtual twin can be used for hands-on experimentation of novice engineering students. Results of the educational study indicated that for students' opinion the workshop effectively exposed them to industrial robotics and was useful and relevant to their future profession. They noted that the exercise on a digital twin of Baxter challenged their spatial reasoning and contributed to the awareness about spatial skills required in operating industrial robots.

\section{Acknowledgement}

This study is supported by the Israel Science Foundation grant.

\section{$7 \quad$ References}

[1] ISO 8373 (2012). https://www.iso.org/standard/55890.html. Retrieved October 28, 2018.

[2] Luo, R. C., \& Kuo, C. W. (2016). Intelligent seven-DoF robot with dynamic obstacle avoidance and 3-D object recognition for industrial cyber-physical systems in manufacturing automation. Proceedings of the IEEE, 104(5), 1102-1113. 
[3] Mäkiö-Marusik, E. (2017). Current trends in teaching cyber physical systems engineering: A literature review. $15^{\text {th }}$ International Conference on Industrial Informatics, Emden, Germany, pp. 518-525. https://doi.org/10.1109/indin.2017.8104826

[4] Cheng, A. M. (2014). An undergraduate cyber-physical systems course. $4^{\text {th }}$ ACM SIGBED International Workshop on Design, Modeling, and Evaluation of Cyber-Physical Systems, Berlin, Germany, pp. 31-34. https://doi.org/10.1145/2593458.2593464

[5] Zalewski, J., \& Gonzalez, F. (2017). Evolution in the education of software engineers: Online course on cyberphysical systems with remote access to robotic devices. International Journal of Online Engineering, 13(8), 133-146. https://doi.org/10.3991/ijoe.v13i08.7377

[6] Crenshaw, T. L. A. (2013). Using robots and contract learning to teach cyber-physical systems to undergraduates. IEEE Transactions on Education, 56(1), 116-120. https://doi.org/10.1109/te.2012.2217967

[7] Meyers, K. L., Ohland, M. W., Pawley, A. L., Silliman, S. E., \& Smith, K. A. (2012). Factors relating to engineering identity. Global Journal of Engineering Education, 14(1), 119131.

[8] Purzer, S., Douglas, K. A., Folkerts, J. A., \& Williams, T. V. (2017). An assessment framework for first-year introduction to engineering courses. 2017 ASEE Annual Conference, Columbus, Ohio. https://doi.org/10.18260/1-2--27552

[9] Crawley, E. F., Malmqvist, J., Östlund S., \& Edström, K. (2014). Rethinking engineering education: The CDIO approach, 2nd ed., New York: Springer. https://doi.org/10.1007/9783-319-05561-9 8

[10] Schuster, K., Groß, K., Vossen, R., Richert, A., \& Jeschke, S. (2016). Preparing for industry 4.0-collaborative virtual learning environments in engineering education. In: Engineering Education 4.0, pp. 477-487. Springer, Cham. https://doi.org/10.1007/978-3-319-46916$\underline{436}$

[11] Karyotaki, M., \& Drigas, A. (2015). Online and other ICT applications for cognitive training and assessment. International Journal of Online Engineering (iJOE), 11(2), 36-42. https://doi.org/10.3991/ijoe.v11i2.4360

[12] Verner, I., \& Gamer, S. (2015). Robotics laboratory classes for spatial training of industrial engineering and vocational school students, International Journal of Engineering Education, 31, pp. 1376-1388. https://doi.org/10.1109/icl.2015.7318216

[13] Fernández-Llamas, C., Conde, M. A., Rodríguez-Lera, F. J., Rodríguez-Sedano, F. J., \& García, F. (2018). May I teach you? Students' behavior when lectured by robotic vs. human teachers. Computers in Human Behavior, 80, 460-469. https://doi.org/10.1016/j.chb.2017.09.028

[14] Jones, J. L., \& Lozano-Perez, T. (1990). Planning two-fingered grasps for pick-and-place operations on polyhedra. 1990 IEEE International Conference on Robotics and Automation, Cincinnati, Ohio, pp. 683-688. https://doi.org/10.1109/robot.1990.126063

[15] Amir, M., Ghalamzan, E., Mavrakis, N., \& Stolkin, R. (2017). Grasp that optimises objectives along post-grasp trajectories. 5th RSI International Conference on Robotics and Mechatronics, Tehran, Iran, pp. 51-56. https://doi.org/10.1109/icrom.2017.8466161

[16] Mavrakis, N., Ghalamzan, E. A. M., \& Stolkin, R. (2017). Safe robotic grasping: Minimum impact-force grasp selection. 2017 IEEE/RSJ International Conference on Robots and Systems, Vancouver, Canada, pp. 4034-4041. https://doi.org/10.1109/iros.2017.8206258 operation skills in a remote robot laboratory. 2018 IEEE Global Engineering Education 
Conference, Santa Cruz de Tenerife, Spain, pp. 389-393. https://doi.org/10.1109/educon.2018.8363256

[18] Kumar, R. R., \& Chand, P. (2015). Inverse kinematics solution for trajectory tracking using artificial neural networks for SCORBOT ER-4u. 6th International Conference on Automation, Robotics and Applications, Queenstown, New Zealand, pp. 364-369. https://doi.org/10.1109/icara.2015.7081175

[19] Baxter workspace guidelines, http://sdk.rethinkrobotics.com/wiki/Workspace Guidelines. Retrieved October 31, 2018.

[20] Baxter arms, http://sdk.rethinkrobotics.com/wiki/Arms. Retrieved October 31, 2018.

\section{Authors}

Igor M. Verner is Professor and Head of the Center for Robotics and Digital Technology Education at the Faculty of Education in Science and Technology, and is also affiliated with the Faculty of Industrial Engineering and Management at Technion - Israel Institute of Technology.

Dan Cuperman is a Ph.D. research associate at the Faculty of Education in Science and Technology, Technion - Israel Institute of Technology.

Sergei Gamer is Director of the Cognitive Robotics Lab of the Faculty of Industrial Engineering and Management at Technion - Israel Institute of Technology.

Alex Polishuk is a Ph.D. research associate at the Faculty of Education in Science and Technology, Technion - Israel Institute of Technology.

Article submitted 2019-03-16. Resubmitted 2019-04-29. Final acceptance 2019-05-04. Final version published as submitted by the authors. 